\title{
Malliavin Differentiability of Solutions of SPDEs with Lévy White Noise
}

\author{
Raluca M. Balan and Cheikh B. Ndongo \\ Department of Mathematics and Statistics, University of Ottawa, 585 King Edward Avenue, Ottawa, ON, Canada K1N 6N5 \\ Correspondence should be addressed to Raluca M. Balan; rbalan@uottawa.ca
}

Received 2 January 2017; Accepted 21 February 2017; Published 12 March 2017

Academic Editor: Bohdan Maslowski

Copyright (C) 2017 Raluca M. Balan and Cheikh B. Ndongo. This is an open access article distributed under the Creative Commons Attribution License, which permits unrestricted use, distribution, and reproduction in any medium, provided the original work is properly cited.

\begin{abstract}
We consider a stochastic partial differential equation (SPDE) driven by a Lévy white noise, with Lipschitz multiplicative term $\sigma$. We prove that, under some conditions, this equation has a unique random field solution. These conditions are verified by the stochastic heat and wave equations. We introduce the basic elements of Malliavin calculus with respect to the compensated Poisson random measure associated with the Lévy white noise. If $\sigma$ is affine, we prove that the solution is Malliavin differentiable and its Malliavin derivative satisfies a stochastic integral equation.
\end{abstract}

\section{Introduction}

In this article, we consider the stochastic partial differential equation (SPDE):

$$
\mathscr{L} u(t, x)=\sigma(u(t, x)) \dot{L}(t, x), \quad t \in[0, T], x \in \mathbb{R},
$$

with some deterministic initial conditions, where $\mathscr{L}$ is a second-order differential operator on $[0, T] \times \mathbb{R}, \dot{L}$ denotes the formal derivative of the Lévy white noise $L$ (defined below), and the function $\sigma: \mathbb{R} \rightarrow \mathbb{R}$ is Lipschitz continuous.

A process $u=\{u(t, x) ; t \in[0, T], x \in \mathbb{R}\}$ is called a (mild) solution of (1) if $u$ is predictable and satisfies the following integral equation:

$$
\begin{aligned}
u(t, x) & \\
= & w(t, x) \\
& \quad+\int_{0}^{t} \int_{\mathbb{R}} G(t-s, x-y) \sigma(u(s, y)) L(d s, d y),
\end{aligned}
$$

where $w$ is the solution of the deterministic equation $\mathscr{L} u=$ 0 with the same initial conditions as (1) and $G$ is the Green function of the operator $\mathscr{L}$.

The study of SPDEs with Gaussian noise is a welldeveloped area of stochastic analysis, and the behaviour of random field solutions of such equations is well understood. We refer the reader to [1] for the original lecture notes which led to the development of this area and to $[2,3]$ for some recent advances. In particular, the probability laws of these solutions can be analyzed using techniques from Malliavin calculus, as described in $[4,5]$.

On the other hand, there is a large literature dedicated to the study of stochastic differential equations (SDE) with Lévy noise, the monograph [6] containing a comprehensive account on this topic. One can develop also a Malliavin calculus for Lévy processes with finite variance, using an analogue of the Wiener chaos representation with respect to underlying Poisson random measure of the Lévy process. This method was developed in [7] with the same purpose of analyzing the probability law of the solution of an SDE driven by a finite variance Lévy noise. More recently, Malliavin calculus for Lévy processes with finite variance has been used in financial mathematics, the monograph [8] being a very readable introduction to this topic.

There are two approaches to SPDEs in the literature. One is the random field approach which originates in John Walsh's lecture notes [1]. When using this approach, the solution is viewed as a real-valued process which is indexed by time and space. The other approach is the infinite-dimensional approach, due to Da Prato and Zabczyk [9], according to 
which the solution is a process indexed by time only, which takes values in an infinite-dimensional Hilbert space. It is not always possible to compare the solutions obtained using the two approaches (see [10] for several results in this direction). SPDEs with Lévy noise were studied in the monograph [11], using the infinite-dimensional approach. In the present article, we use the random field approach for examining an SPDE driven by the finite variance Lévy noise introduced in [12], with the goal of studying the Malliavin differentiability of the solution. As mentioned above, this study can be useful for analyzing the probability law of the solution. We postpone this problem for future work.

We begin by recalling from [12] the construction of the Lévy white noise $L$ driving (1). We consider a Poisson random measure $(\mathrm{PRM}) N$ on the space $U=[0, T] \times \mathbb{R} \times \mathbb{R}_{0}$ of intensity $\mu(d t, d x, d z)=d t d x \nu(d z)$ defined on a complete probability space $(\Omega, \mathscr{F}, P)$, where $v$ is a Lévy measure on $\mathbb{R}_{0}=\mathbb{R} \backslash\{0\}$; that is, $v$ satisfies

$$
\int_{\mathbb{R}_{0}}\left(1 \wedge|z|^{2}\right) \nu(d z)<\infty
$$

In addition, we assume that $\nu$ satisfies the following condition:

$$
v:=\int_{\mathbb{R}_{0}} z^{2} v(d z)<\infty
$$

We denote by $\widehat{N}$ the compensated PRM defined by $\widehat{N}(A)=N(A)-\mu(A)$ for any $A \in \mathcal{U}$ with $\mu(A)<\infty$, where $\mathcal{U}$ is the class of Borel sets in $U$. We denote by $\mathscr{F}_{t}$ the $\sigma$-field generated by $N([0, s] \times B \times \Gamma)$ for all $s \in[0, t], B \in \mathscr{B}_{b}(\mathbb{R})$, and $\Gamma \in \mathscr{B}_{b}\left(\mathbb{R}_{0}\right)$. We denote by $\mathscr{B}_{b}(\mathbb{R})$ the class of bounded Borel sets in $\mathbb{R}$ and by $\mathscr{B}_{b}\left(\mathbb{R}_{0}\right)$ the class of Borel sets in $\mathbb{R}_{0}$ which are bounded away from 0 .

A Lévy white noise with intensity measure $v$ is a collection $L=\left\{L_{t}(B) ; t \in[0, T], B \in \mathscr{B}_{b}(\mathbb{R})\right\}$ of zero-mean squareintegrable random variables defined by

$$
L_{t}(B)=\int_{0}^{t} \int_{B} \int_{\mathbb{R}_{0}} z \widehat{N}(d s, d x, d z)
$$

These variables have the following properties:

(i) $L_{0}(B)=0$ a.s. for all $B \in \mathscr{B}_{b}(\mathbb{R})$.

(ii) $L_{t}\left(B_{1}\right), \ldots, L_{t}\left(B_{k}\right)$ are independent for any $t>0$ and for any disjoint sets $B_{1}, \ldots, B_{k} \in \mathscr{B}_{b}(\mathbb{R})$.

(iii) For any $0<s \leq t$ and for any $B \in \mathscr{B}_{b}(\mathbb{R}), L_{t}(B)-$ $L_{s}(B)$ is independent of $\mathscr{F}_{s}$ and has characteristic function

$$
\begin{aligned}
& E\left(e^{i u\left(L_{t}(B)-L_{s}(B)\right)}\right) \\
& \quad=\exp \left\{(t-s)|B| \int_{\mathbb{R}_{0}}\left(e^{i u z}-1-i u z\right) \nu(d z)\right\},
\end{aligned}
$$

We denote by $\mathscr{F}_{t}^{L}$ the $\sigma$-field generated by $L(s)$ for all $s \in[0, t]$. For any $h \in L^{2}([0, T] \times \mathbb{R})$, we define the stochastic integral of $h$ with respect to $L$ :

$$
\begin{aligned}
L(h) & =\int_{0}^{T} \int_{\mathbb{R}} h(t, x) L(d t, d x) \\
& =\int_{0}^{T} \int_{\mathbb{R}} \int_{\mathbb{R}_{0}} h(t, x) z \widehat{N}(d t, d x, d z) .
\end{aligned}
$$

Using the same method as in Itô's classical theory, this integral can be extended to random integrands, that is, to the class of predictable processes $X=\{X(t, x) ; t \in[0, T], x \in$ $\mathbb{R}\}$, such that $E \int_{0}^{T} \int_{\mathbb{R}}|X(t, x)|^{2} d x d t<\infty$. The integral has the following isometry property:

$$
\begin{aligned}
& E\left|\int_{0}^{T} \int_{\mathbb{R}} X(t, x) L(d t, d x)\right|^{2} \\
& \quad=v E \int_{0}^{T} \int_{\mathbb{R}}|X(t, x)|^{2} d x d t .
\end{aligned}
$$

Recall that a process $X=\left\{X(t, x) ; t \geq 0, x \in \mathbb{R}^{d}\right\}$ is predictable if it is measurable with respect to the predictable $\sigma$-field on $\Omega \times \mathbb{R}_{+} \times \mathbb{R}$, that is, the $\sigma$-field generated by elementary processes of the form

$$
\begin{aligned}
X(\omega, t, x)=Y(\omega) 1_{(a, b]}(t) 1_{A}(x), & \\
& \omega \in \Omega, t \in[0, T], x \in \mathbb{R},
\end{aligned}
$$

where $0<a<b, Y$ is a bounded and $\mathscr{F}_{a}^{L}$-measurable random variable, and $A \in \mathscr{B}_{b}(\mathbb{R})$.

This article is organized as follows. In Section 2, we introduce the basic elements of Malliavin calculus with respect to the compensated Poisson random measure $\widehat{N}$. In Section 3, we prove that, under a certain hypothesis, (1) has a unique solution. This hypothesis is verified in the case of the wave and heat equations. In Section 4, we examine the Malliavin differentiability of the solution, in the case when the function $\sigma$ is affine. Finally, in the Appendix, we include a version of Gronwall's lemma which is needed in the sequel.

\section{Malliavin Calculus on the Poisson Space}

In this section, we introduce the basic ingredients of Malliavin calculus with respect to $\widehat{N}$, following very closely the approach presented in Chapters 10-12 of [8]. The difference compared to [8] is that our parameter space $U$ has variables $(t, x, z)$ instead of $(t, z)$. For the sake of brevity, we do not include the proofs of the results presented in this section. These proofs can be found in Chapter 6 of the doctoral thesis [13] of the second author.

We set $\mathscr{H}=L^{2}(U, \mathcal{U}, \mu)$ and $\mathscr{H}^{\otimes n}=L^{2}\left(U^{n}, \mathscr{U}^{n}, \mu^{n}\right)$. We denote by $\mathscr{H}^{\odot}$ the set of all symmetric functions $f \in \mathscr{H}^{\otimes n}$. We denote by $\mathscr{H}_{\mathbb{C}}, \mathscr{H}_{\mathbb{C}}^{\otimes n}, \mathscr{H}_{\mathbb{C}}^{\odot n}$ the analogous spaces of $\mathbb{C}$-valued functions. 
Let $S_{n}=\left\{\left(u_{1}, \ldots, u_{n}\right) \in U^{n} ; u_{i}=\left(t_{i}, x_{i}, z_{i}\right)\right.$ with $t_{1}<$ $\left.\cdots<t_{n}\right\}$. For any measurable function $f: S_{n} \rightarrow \mathbb{R}$ with

$$
\begin{aligned}
\|f\|_{L^{2}\left(S_{n}\right)} & :=\int_{S_{n}}\left|f\left(u_{1}, \ldots, u_{n}\right)\right|^{2} d \mu^{n}\left(u_{1}, \ldots, u_{n}\right) \\
& <\infty,
\end{aligned}
$$

we define the $n$-fold iterated integral of $f$ with respect to $\widehat{N}$ by

$$
\begin{aligned}
& J_{n}(f) \\
& \quad=\int_{0}^{T} \int_{\mathbb{R}} \int_{\mathbb{R}_{0}} \ldots\left(\int_{0}^{t_{2}-} \int_{\mathbb{R}} \int_{\mathbb{R}_{0}} f\left(u_{1}, \ldots, u_{n}\right) \widehat{N}\left(d u_{1}\right)\right) \\
& \quad \ldots \widehat{N}\left(d u_{n}\right),
\end{aligned}
$$

where $u_{i}=\left(t_{i}, x_{i}, z_{i}\right)$. Then, $E\left[J_{n}(f) J_{m}(g)\right]=0$ for all $n \neq m$ and $E\left|J_{n}(f)\right|^{2}=\|f\|_{L^{2}\left(S_{n}\right)}^{2}$.

For any $f \in \mathscr{H}^{\odot n}$, we defined the multiple integral of $f$ with respect to $\widehat{N}$ by $I_{n}(f)=n ! J_{n}(f)$. It follows that $E\left[I_{n}(f) I_{m}(g)\right]=0$ for all $n \neq m$ and

$$
E\left|I_{n}(f)\right|^{2}=n !\|f\|_{\mathscr{H}^{\otimes n}}^{2} \quad \forall f \in \mathscr{H}^{\odot n} .
$$

If $f \in \mathscr{H}_{\mathbb{C}}^{\odot n}$ with $f=g+i h$, we define $I_{n}(f)=I_{n}(g)+i I_{n}(h)$.

Let $L_{\mathbb{C}}^{2}(\Omega)$ be the set of $\mathbb{C}$-valued square-integrable random variables defined on $(\Omega, \mathscr{F}, P)$. By Theorem 7 of [14], any $\mathscr{F}_{T}^{L}$-measurable random variable $F \in L_{\mathbb{C}}^{2}(\Omega)$ admits the chaos expansion

$$
F=\sum_{n \geq 0} I_{n}\left(f_{n}\right) \quad \text { in } L_{\mathbb{C}}^{2}(\Omega),
$$

where $f_{n} \in \mathscr{H}^{\odot n}$ for all $n \geq 1$ and $f_{0}=E(F)$.

The chaos expansion plays a crucial role in developing the Malliavin calculus with respect to $\widehat{N}$. In particular, the Skorohod integrals with respect to $\widehat{N}$ and $L$ are defined as follows.

Definition 1. (a) Let $X=\{X(u) ; u \in U\}$ be a squareintegrable process such that $X(u)$ is $\mathscr{F}_{T}^{L}$-measurable for any $u \in U$. For each $u \in U$, let $X(u)=\sum_{n \geq 0} I_{n}\left(f_{n}(\cdot, u)\right)$ be the chaos expansion of $X(u)$, with $f_{n}(\cdot, u) \in \mathscr{H}^{\circ n}$. One denotes by $\widetilde{f}_{n}\left(u_{1}, \ldots, u_{n}, u\right)$ the symmetrization of $f_{n}$ with respect to all $n+1$ variables. One says that $X$ is Skorohod integrable with respect to $\widehat{N}$ (and one writes $X \in \operatorname{Dom}(\delta)$ ) if

$$
\sum_{n \geq 0} E\left|I_{n+1}\left(\tilde{f}_{n}\right)\right|^{2}=\sum_{n \geq 0}(n+1) !\left\|\tilde{f}_{n}\right\|_{\mathscr{H}^{\odot}(n+1)}^{2}<\infty .
$$

In this case, one defines the Skorohod integral of $X$ with respect to $\widehat{N}$ by

$$
\begin{aligned}
\delta(X) & =\int_{0}^{T} \int_{\mathbb{R}} \int_{\mathbb{R}_{0}} X(t, x, z) \widehat{N}(\delta t, \delta x, \delta z) \\
& :=\sum_{n \geq 0} I_{n+1}\left(\widetilde{f}_{n}\right) .
\end{aligned}
$$

(b) Let $Y=\{Y(t, x) ; t \in[0, T], x \in \mathbb{R}\}$ be a squareintegrable process such that $Y(t, x)$ is $\mathscr{F}_{T}^{L}$-measurable for any $t \in[0, T]$ and $x \in \mathbb{R}$. One says that $Y$ is Skorohod integrable with respect to $L$ (and one writes $Y \in \operatorname{Dom}\left(\delta^{L}\right)$ ) if the process $\{Y(t, x) z ;(t, x, z) \in U\}$ is Skorohod integrable with respect to $\widehat{N}$. In this case, one defines the Skorohod integral of $Y$ with respect to $L$ by

$$
\begin{aligned}
\delta^{L}(Y) & =\int_{0}^{T} \int_{\mathbb{R}} Y(t, x) L(\delta t, \delta x) \\
& :=\int_{0}^{T} \int_{\mathbb{R}} \int_{\mathbb{R}_{0}} Y(t, x) z \widehat{N}(\delta t, \delta x, \delta z) .
\end{aligned}
$$

The following result shows that the Skorohod integral can be viewed as an extension of the Itô integral.

Theorem 2. (a) If $X=\{X(u) ;(u) \in U\}$ is a predictable process such that $E\|X\|_{U}^{2}<\infty$, then $X$ is Skorohod integrable with respect to $\widehat{N}$ and

$$
\begin{aligned}
& \int_{0}^{T} \int_{\mathbb{R}} \int_{\mathbb{R}_{0}} X(t, x, z) \widehat{N}(\delta t, \delta x, \delta z) \\
& \quad=\int_{0}^{T} \int_{\mathbb{R}} \int_{\mathbb{R}_{0}} X(t, x, z) \widehat{N}(d t, d x, d z) .
\end{aligned}
$$

(b) If $Y=\{Y(t, x) ; t \in[0, T], x \in \mathbb{R}\}$ is a predictable process such that $E \int_{0}^{T} \int_{\mathbb{R}}|Y(t, x)|^{2} d x d t<\infty$, then $Y$ is Skorohod integrable with respect to $L$ and

$$
\int_{0}^{T} \int_{\mathbb{R}} Y(t, x) L(\delta t, \delta x)=\int_{0}^{T} \int_{\mathbb{R}} Y(t, x) L(d t, d x) .
$$

We now introduce the definition of the Malliavin derivative.

Definition 3. Let $F \in L^{2}(\Omega)$ be an $\mathscr{F}_{T}^{L}$-measurable random variable with the chaos expansion $F=\sum_{n \geq 0} I_{n}\left(f_{n}\right)$ with $f_{n} \epsilon$ $\mathscr{H}^{\odot n}$. One says that $F$ is Malliavin differentiable with respect to $\widehat{N}$ if

$$
\sum_{n \geq 1} n n !\left\|f_{n}\right\|_{\mathscr{H}^{\otimes n}}^{2}<\infty
$$

In this case, one defines the Malliavin derivative of $F$ with respect to $\widehat{N}$ by

$$
D_{u} F=\sum_{n \geq 1} n I_{n-1}\left(f_{n}(\cdot, u)\right), \quad u \in U .
$$

One denotes by $\mathbb{D}^{1,2}$ the space of Malliavin differentiable random variables with respect to $\widehat{N}$.

$$
\text { Note that } E\|D F\|_{\mathscr{H}}^{2}=\sum_{n \geq 1} n n !\left\|f_{n}\right\|_{\mathscr{H}^{\otimes n}}^{2}<\infty \text {. }
$$

Theorem 4 (closability of Malliavin derivative). Let $\left(F_{n}\right)_{n \geq 1} \subset$ $\mathbb{D}^{1,2}$ and $F \in L^{2}(\Omega)$ such that $F_{n} \rightarrow F$ in $L^{2}(\Omega)$ and $\left(D F_{n}\right)_{n \geq 1}$ converges in $L^{2}(\Omega ; \mathscr{H})$. Then, $F \in \mathbb{D}^{1,2}$ and $D F_{n} \rightarrow D F$ in $L^{2}(\Omega ; \mathscr{H})$. 
Typical examples of Malliavin differentiable random variables are exponentials of stochastic integrals: for any $h \in$ $L^{2}([0, T] \times \mathbb{R})$,

$$
D_{t, x, z}\left(e^{L(h)}\right)=e^{L(h)}\left(e^{h(t, x) z}-1\right) .
$$

Moreover, the set $\mathbb{D}_{\mathscr{G}}^{1,2}$ of linear combinations of random variables of the form $e^{L(h)}$ with $h \in L^{2}([0, T] \times \mathbb{R})$ is dense in $\mathbb{D}^{1,2}$.

The following result shows that the Malliavin derivative is a difference operator with respect to $\widehat{N}$, not a differential operator.

Theorem 5 (chain rule). For any $F \in \mathbb{D}^{1,2}$ and any continuous function $g: \mathbb{R} \rightarrow \mathbb{R}$ such that $g(F) \in L^{2}(\Omega)$ and $g(F+D F)$ $g(F) \in L^{2}(\Omega ; \mathscr{H}), g(F) \in \mathbb{D}^{1,2}$ and

$$
D g(F)=g(F+D F)-g(F) \quad \text { in } L^{2}(\Omega ; \mathscr{H}) .
$$

Similar to the Gaussian case, we have the following results.

Theorem 6 (duality formula). If $F \in \mathbb{D}^{1,2}$ and $X \in \operatorname{Dom}(\delta)$, then

$$
\begin{aligned}
E & {\left[F \int_{0}^{T} \int_{\mathbb{R}} \int_{\mathbb{R}_{0}} X(t, x, z) \widehat{N}(\delta t, \delta x, \delta z)\right] } \\
& =E\left[\int_{0}^{T} \int_{\mathbb{R}_{0}} \int_{\mathbb{R}_{0}} X(t, x, z) D_{t, x, z} F \nu(d z) d x d t\right] .
\end{aligned}
$$

Theorem 7 (fundamental theorem of calculus). Let $X=$ $\left\{X(s, y, \zeta) ; s \in[0, T], y \in \mathbb{R}, \zeta \in \mathbb{R}_{0}\right\}$ be a process which satisfies the following conditions:

(i) $X(s, y, \zeta) \in \mathbb{D}^{1,2}$ for any $(s, y, \zeta) \in U$.

(ii) $E \int_{0}^{T} \int_{\mathbb{R}} \int_{\mathbb{R}_{0}}|X(s, y, \zeta)|^{2} \nu(d z) d y d s<\infty$.

(iii) $\left\{D_{t, x, z} X(s, y, \zeta) ;(s, y, \zeta) \in U\right\} \in \operatorname{Dom}(\delta)$ for any $(t, x, z) \in U$.

(iv) $\left\{\delta\left(D_{t, x, z} X\right) ;(t, x, z) \in U\right\} \in L^{2}(\Omega ; \mathscr{H})$.

Then, $X \in \operatorname{Dom}(\delta), \delta(X) \in \mathbb{D}^{1,2}$ and $D[\delta(X)]=X+$ $\delta(D X)$; that is,

$$
\begin{aligned}
& D_{t, x, z}\left(\int_{0}^{T} \int_{\mathbb{R}} \int_{\mathbb{R}_{0}} X(s, y, \zeta) \widehat{N}(\delta s, \delta y, \delta \zeta)\right) \\
&=X(t, x, z) \\
& \quad+\int_{0}^{T} \int_{\mathbb{R}} \int_{\mathbb{R}_{0}} D_{t, x, z} X(s, y, \zeta) \widehat{N}(\delta s, \delta y, \delta \zeta)
\end{aligned}
$$

$$
\text { in } L^{2}(\Omega ; \mathscr{H}) \text {. }
$$

As an immediate consequence of the previous theorem, we obtain the following result.
Theorem 8. Let $Y=\{Y(s, y) ; s \in[0, T], y \in \mathbb{R}\}$ be a process which satisfies the following conditions:

(i) $Y(s, y) \in \mathbb{D}^{1,2}$ for all $s \in[0, T]$ and $y \in \mathbb{R}$.

(ii) $E \int_{0}^{T} \int_{\mathbb{R}}|Y(s, y)|^{2} d y d s<\infty$.

(iii) $\left\{D_{t, x, z} Y(s, y) ; s \in[0, T], y \in \mathbb{R}\right\} \in \operatorname{Dom}\left(\delta^{L}\right)$ for any $(t, x, z) \in U$.

(iv) $E \int_{0}^{T} \int_{\mathbb{R}} \int_{\mathbb{R}_{0}}\left|\int_{0}^{T} \int_{\mathbb{R}} D_{t, x, z} Y(s, y) L(\delta s, \delta y)\right|^{2} v(d z) d x d t$ $<\infty$.

Then, $Y \in \operatorname{Dom}\left(\delta^{L}\right), \delta^{L}(Y) \in \mathbb{D}^{1,2}$ and the following relation holds in $L^{2}(\Omega ; \mathscr{H})$ :

$$
\begin{aligned}
& D_{t, x, z}\left(\delta^{L}(Y)\right) \\
& \quad=Y(t, x) z+\int_{0}^{T} \int_{\mathbb{R}} \int_{\mathbb{R}_{0}} D_{t, x, z} Y(s, y) L(\delta s, \delta y) .
\end{aligned}
$$

\section{Existence of Solution}

In this section, we show that (1) has a unique solution.

We recall that $w$ is the solution of the homogeneous equation $\mathscr{L} u=0$ with the same initial conditions as (1), and $G$ is the Green function of the operator $\mathscr{L}$ on $\mathbb{R}_{+} \times \mathbb{R}$. We assume that, for any $t \in[0, T], G(t, \cdot) \in L^{1}(\mathbb{R})$ and we denote by $\mathscr{F} G(t, \cdot)$ its Fourier transform:

$$
\mathscr{F} G(t, \cdot)(\xi)=\int_{\mathbb{R}} e^{-i \xi x} G(t, x) d x .
$$

We suppose that the following hypotheses hold.

Hypothesis H1. $w$ is continuous and uniformly bounded on $[0, T] \times \mathbb{R}$.

Hypothesis H2. (a) $\int_{0}^{T} \int_{\mathbb{R}} G^{2}(t, x) d x d t<\infty$; (b) the function $t \mapsto \mathscr{F} G(t, \cdot)(\xi)$ is continuous on $[0, T]$, for any $\xi \in \mathbb{R}^{d}$; (c) there exist $\varepsilon>0$ and a nonnegative function $k_{t}(\cdot)$ such that

$$
|\mathscr{F} G(t+h, \cdot)(\xi)-\mathscr{F} G(t, \cdot)(\xi)| \leq k_{t}(\xi)
$$

for any $t \in[0, T]$ and $h \in[0, \varepsilon]$, and $\int_{0}^{T} \int_{\mathbb{R}}\left|k_{t}(\xi)\right|^{2} d \xi d t<\infty$.

Since $\sigma$ is a Lipschitz continuous function, there exists a constant $C_{\sigma}>0$ such that, for any $x, y \in \mathbb{R}$,

$$
|\sigma(x)-\sigma(y)| \leq C_{\sigma}|x-y| .
$$

In particular, for any $x \in \mathbb{R}$,

$$
|\sigma(x)| \leq D_{\sigma}(1+|x|),
$$

where $D_{\sigma}=\max \left\{C_{\sigma},|\sigma(0)|\right\}$.

The following theorem is an extension of Theorem 1.1.(a) of [15] to an arbitrary operator $\mathscr{L}$.

Theorem 9. Equation (1) has a unique solution $u=\{u(t, x)$; $t \in[0, T], x \in \mathbb{R}\}$ which is $L^{2}(\Omega)$-continuous and satisfies

$$
\sup _{(t, x) \in[0, T] \times \mathbb{R}} E|u(t, x)|^{2}<\infty .
$$


Proof.

Existence. We use the same argument as in the proof of Theorem 13 of [16]. We denote by $\left(u_{n}\right)_{n \geq 0}$ the sequence of Picard iterations defined by $u_{0}(t, x)=w(t, x)$ and

$$
\begin{aligned}
& u_{n+1}(t, x) \\
& =w(t, x) \\
& \quad+\int_{0}^{t} \int_{\mathbb{R}} G(t-s, x-y) \sigma\left(u_{n}(s, y)\right) L(d s, d y),
\end{aligned}
$$

$$
n \geq 0 \text {. }
$$

By induction on $n$, it can be proved that the following properties hold:

(P)

(i) $u_{n}(t, x)$ is well defined for any $(t, x) \in[0, T] \times \mathbb{R}$.

(ii) $K_{n}:=\sup _{(t, x) \in[0, T] \times \mathbb{R}} E\left|u_{n}(t, x)\right|^{2}<\infty$.

(iii) $(t, x) \mapsto u_{n}(t, x)$ is $L^{2}(\Omega)$-continuous on $[0, T] \times$ $\mathbb{R}$.

(iv) $u_{n}(t, x)$ is $\mathscr{F}_{t}$-measurable for any $t \in[0, T]$ and $x \in \mathbb{R}$.

Hypotheses ( $\mathrm{H} 1)$ and $(\mathrm{H} 2)$ are needed for the proof of property (iii). From properties (iii) and (iv), it follows that $u_{n}$ has a predictable modification, denoted also by $u_{n}$. This modification is used in definition (31) of $u_{n+1}(t, x)$. Using the isometry property (8) of the stochastic integral and (28), we have

$$
\begin{aligned}
& E\left|u_{n+1}(t, x)-u_{n}(t, x)\right|^{2}=v E \int_{0}^{t} \int_{\mathbb{R}} G^{2}(t-s, x-y) \\
& \cdot\left|\sigma\left(u_{n}(s, y)\right)-\sigma\left(u_{n-1}(s, y)\right)\right|^{2} d y d s \\
& \leq v C_{\sigma}^{2} \int_{0}^{t} \int_{\mathbb{R}} G^{2}(t-s, x-y) \\
& \cdot E\left|u_{n}(s, y)-u_{n-1}(s, y)\right|^{2} d y d s \\
& \leq v C_{\sigma}^{2} \int_{0}^{t} H_{n}(s)\left(\int_{\mathbb{R}} G^{2}(t-s, x-y) d y\right) d s
\end{aligned}
$$

where $H_{n}(t)=\sup _{x \in \mathbb{R}} E\left|u_{n}(t, x)-u_{n-1}(t, x)\right|^{2}$. For any $t \in$ $[0, T]$, we denote

$$
J(t)=\int_{\mathbb{R}} G^{2}(t, x) d x .
$$

Taking the supremum over $x \in \mathbb{R}$ in the previous inequality, we obtain that

$$
H_{n+1}(t) \leq v C_{\sigma}^{2} \int_{0}^{t} H_{n}(s) J(t-s) d s,
$$

for any $t \in[0, T]$ and $n \geq 0$. By applying Lemma A.1 (the Appendix) with $C_{n}=0$ and $p=2$, we infer that

$$
\sum_{n \geq 0} \sup _{t \in[0, T]} H_{n}(t)^{1 / 2}<\infty .
$$

This shows that the sequence $\left(u_{n}\right)_{n \geq 0}$ converges in $L^{2}(\Omega)$ to a random variable $u(t, x)$, uniformly in $[0, T] \times \mathbb{R}$; that is,

$$
\sup _{(t, x) \in[0, T] \times \mathbb{R}} E\left|u_{n}(t, x)-u(t, x)\right|^{2} \longrightarrow 0 .
$$

To see that $u$ is a solution of (1), we take the limit in $L^{2}(\Omega)$ as $n \rightarrow \infty$ in (31). In particular, this argument shows that

$$
K:=\sup _{n \geq 1} \sup _{(t, x) \in[0, T] \times \mathbb{R}} E\left|u_{n}(t, x)\right|^{2}<\infty .
$$

Uniqueness. Let $H(t)=\sup _{x \in \mathbb{R}} E\left|u(t, x)-u^{\prime}(t, x)\right|^{2}$, where $u$ and $u^{\prime}$ are two solutions of (1). A similar argument as above shows that

$$
H(t) \leq v C_{\sigma}^{2} \int_{0}^{t} H(s) J(t-s) d s,
$$

for any $t \in[0, T]$. By Gronwall's lemma, $H(t)=0$ for all $t \in$ $[0, T]$.

Example 10 (wave equation). If $\mathscr{L} u=\partial u / \partial^{2} t-\partial u / \partial x^{2}$ for $t \in[0, T]$ and $x \in \mathbb{R}$, then $G(t, x)=(1 / 2) 1_{\{|x| \leq t\}}$. Hypothesis (H2) holds since

$$
\mathscr{F} G(t, \cdot)(\xi)=\frac{\sin (t|\xi|)}{|\xi|} .
$$

Example 11 (heat equation). If $\mathscr{L} u=\partial u / \partial t-(1 / 2)\left(\partial u / \partial x^{2}\right)$ for $t \in[0, T]$ and $x \in \mathbb{R}$, then $G(t, x)=(2 \pi t)^{-1 / 2} \exp \left(-|x|^{2} /\right.$ $2 t)$. Hypothesis (H2) holds since

$$
\mathscr{F} G(t, \cdot)(\xi)=\exp \left(-\frac{t|\xi|^{2}}{2}\right) .
$$

\section{Malliavin Differentiability of the Solution}

In this section, we show that the solution of (1) is Malliavin differentiable and its Malliavin derivative satisfies a certain integral equation. For this, we assume that the function $\sigma$ is affine.

Our first result shows that the sequence of Picard iterations is Malliavin differentiable with respect to $\widehat{N}$ and the corresponding sequence of Malliavin derivatives is uniformly bounded in $L^{2}(\Omega ; \mathscr{H})$.

Lemma 12. Assume that $\sigma$ is an arbitrary Lipschitz function. Let $\left(u_{n}\right)_{n \geq 0}$ be the sequence of Picard iterations defined by (31). Then, $u_{n}(t, x) \in \mathbb{D}^{1,2}$ for any $(t, x) \in[0, T] \times \mathbb{R}$ and $n \geq 0$, and

$$
A:=\sup _{n \geq 0} \sup _{(t, x) \in[0, T] \times \mathbb{R}} E\left\|D u_{n}(t, x)\right\|_{\mathscr{H}}^{2}<\infty .
$$

Proof.

Step 1. We prove that the following property holds for any $n \geq$ 0 :

$$
\begin{gathered}
u_{n}(t, x) \in \mathbb{D}^{1,2} \text { for any }(t, x) \in[0, T] \times \mathbb{R}, \\
A_{n}:=\sup _{(t, x) \in[0, T] \times \mathbb{R}} E\left\|D u_{n}(t, x)\right\|_{\mathscr{H}}^{2}<\infty .
\end{gathered}
$$


For this, we use an induction argument on $n$. Property (Q) is clear for $n=0$. We assume that it holds for $n$ and we prove that it holds for $n+1$.

By the definition of $u_{n+1}$ and the fact that the Itô integral coincides with the Skorohod integral if the integrand is predictable, it follows that, for any $(t, x) \in[0, T] \times \mathbb{R}$,

$$
\begin{aligned}
& u_{n+1}(t, x) \\
& =w(t, x) \\
& \quad+\int_{0}^{t} \int_{\mathbb{R}} G(t-s, x-y) \sigma\left(u_{n}(s, y)\right) L(\delta s, \delta y) .
\end{aligned}
$$

We fix $(t, x) \in[0, T] \times \mathbb{R}$. We apply the fundamental theorem of calculus for the Skorohod integral with respect to $L$ (Theorem 8 ) to the process:

$$
\begin{aligned}
Y(s, y)=G(t-s, x-y) \sigma\left(u_{n}(s, y)\right) 1_{[0, t]}(s), & \\
& s \in[0, T], y \in \mathbb{R} .
\end{aligned}
$$

We need to check that $Y$ satisfies the hypotheses of this theorem. To check that $Y$ satisfies (i), we apply the chain rule (Theorem 5) to $F=u_{n}(s, y)$ and $g=\sigma$. Note that, for any $(s, y) \in[0, T] \times \mathbb{R}$,

$$
\begin{aligned}
& E\left|\sigma\left(u_{n}(s, y)\right)\right|^{2} \leq 2 D_{\sigma}^{2}\left(1+E\left|u_{n}(s, y)\right|^{2}\right) \leq 2 D_{\sigma}^{2}\left(1+K_{n}\right) \\
& <\infty, \\
& E \int_{0}^{T} \int_{\mathbb{R}} \int_{\mathbb{R}_{0}}\left|\sigma\left(u_{n}(s, y)+D_{r, \xi, z} u_{n}(s, y)\right)-\sigma\left(u_{n}(s, y)\right)\right|^{2} \\
& \quad \cdot(d z) d \xi d r \leq C_{\sigma}^{2} E \int_{0}^{T} \int_{\mathbb{R}} \int_{\mathbb{R}_{0}}\left|D_{r, \xi, z} u_{n}(s, y)\right|^{2} \\
& \cdot \nu(d z) d \xi d r \leq C_{\sigma}^{2} A_{n}<\infty,
\end{aligned}
$$

by the induction hypothesis. We conclude that $Y(s, y) \in \mathbb{D}^{1,2}$ and

$$
\begin{aligned}
D_{r, \xi, z} Y(s, y)=G(t-s, x-y) \\
\cdot\left[\sigma\left(u_{n}(s, y)+D_{r, \xi, z} u_{n}(s, y)\right)-\sigma\left(u_{n}(s, y)\right)\right] \\
\cdot 1_{[0, t]}(s) .
\end{aligned}
$$

We note that $Y$ satisfies hypothesis (ii) since, by (44),

$$
\begin{aligned}
& E \int_{0}^{T} \int_{\mathbb{R}}|Y(s, y)|^{2} d y d s \\
& \quad \leq 2 D_{\sigma}^{2}\left(1+K_{n}\right) \int_{0}^{t} \int_{\mathbb{R}} G^{2}(t-s, x-y) d y d s \\
& \quad<\infty .
\end{aligned}
$$

To check that $Y$ satisfies hypothesis (iii), i.e., the process $\left\{D_{r, \xi, z} Y(s, y) ; s \in[0, T], y \in \mathbb{R}\right\}$ is Skorohod integrable with respect to $L$ for any $(r, \xi, z) \in U$, it suffices to show that this process is Itô integrable with respect to $L$. Note that $D_{r, \xi, z} u_{n}(s, y)=0$ if $r>s$ and it is $\mathscr{F}_{s}$-measurable if $r \leq s$.
Hence, the process $\left\{D_{r, \xi, z} Y(s, y) ; s \in[0, T], y \in \mathbb{R}\right\}$ is predictable. By (46) and (28),

$$
\begin{aligned}
& E \int_{0}^{T} \int_{\mathbb{R}}\left|D_{r, \xi, z} Y(s, y)\right|^{2} d y d s \\
& \leq C_{\sigma}^{2} E \int_{0}^{t} \int_{\mathbb{R}} G^{2}(t-s, x-y)\left|D_{r, \xi, z} u_{n}(s, y)\right|^{2} d y d s,
\end{aligned}
$$

and, hence,

$$
\begin{aligned}
& \int_{0}^{T} \int_{\mathbb{R}} \int_{\mathbb{R}_{0}}\left(E \int_{0}^{T} \int_{\mathbb{R}}\left|D_{r, \xi, z} Y(s, y)\right|^{2} d y d s\right) \\
& \cdot \nu(d z) d \xi d r \leq C_{\sigma}^{2} \int_{0}^{t} \int_{\mathbb{R}} G^{2}(t-s, x-y) \\
& \cdot E\left\|D u_{n}(s, y)\right\|_{\mathscr{H}}^{2} d y d s \\
& \leq C_{\sigma}^{2} A_{n} \int_{0}^{t} \int_{\mathbb{R}} G^{2}(t-s, x-y) d y d s<\infty .
\end{aligned}
$$

This proves that $E \int_{0}^{T} \int_{\mathbb{R}}\left|D_{r, \xi, z} Y(s, y)\right|^{2} d y d s<\infty$ for almost all $(r, \xi, z) \in[0, T] \times \mathbb{R} \times \mathbb{R}_{0}$. By Theorem $2(\mathrm{~b}),\left\{D_{r, \xi, z} Y(s\right.$, $y) ; s \in[0, T], y \in \mathbb{R}\}$ is Skorohod integrable with respect to $L$ and

$$
\begin{aligned}
& \int_{0}^{T} \int_{\mathbb{R}} D_{r, \xi, z} Y(s, y) L(\delta s, \delta y) \\
& \quad=\int_{0}^{T} \int_{\mathbb{R}} D_{r, \xi, z} Y(s, y) L(d s, d y) .
\end{aligned}
$$

Finally, $Y$ satisfies hypothesis (iv) since, by (50) and the isometry properties (8) and (49), we have

$$
\begin{aligned}
& E \int_{0}^{T} \int_{\mathbb{R}} \int_{\mathbb{R}_{0}}\left|\int_{0}^{T} \int_{\mathbb{R}} \int_{\mathbb{R}_{0}} D_{r, \xi, z} Y(s, y) L(\delta s, \delta y)\right|^{2} \\
& \cdot \nu(d z) d \xi d r \\
& =E \int_{0}^{T} \int_{\mathbb{R}} \int_{\mathbb{R}_{0}}\left|\int_{0}^{T} \int_{\mathbb{R}} \int_{\mathbb{R}_{0}} D_{r, \xi, z} Y(s, y) L(d s, d y)\right|^{2} \\
& \cdot \nu(d z) d \xi d r \\
& \quad=v \int_{0}^{T} \int_{\mathbb{R}} \int_{\mathbb{R}_{0}}\left(E \int_{0}^{T} \int_{\mathbb{R}} \int_{\mathbb{R}_{0}}\left|D_{r, \xi, z} Y(s, y)\right|^{2} d y d s\right) \\
& \quad \cdot v(d z) d \xi d r<\infty .
\end{aligned}
$$
and

By Theorem 8, we infer that $Y \in \operatorname{Dom}\left(\delta^{L}\right), \delta^{L}(Y) \in \mathbb{D}^{1,2}$,

$$
\begin{aligned}
D_{r, \xi, z}\left(\delta^{L}(Y)\right)= & Y(r, \xi) z \\
& +\int_{0}^{t} \int_{\mathbb{R}} D_{r, \xi, z} Y(s, y) L(\delta s, \delta y) .
\end{aligned}
$$


Since $u_{n+1}(t, x)=w(t, x)+\delta^{L}(Y)$, this means that $u_{n+1}(t, x) \in$ $\mathbb{D}^{1,2}$. Using (50) and (46), we can rewrite relation (52) as follows:

$$
\begin{aligned}
D_{r, \xi, z} u_{n+1}(t, x)=G(t-r, x-\xi) \sigma\left(u_{n}(r, \xi)\right) z \\
+\int_{0}^{t} \int_{\mathbb{R}} G(t-s, x-y) \\
\cdot\left[\sigma\left(u_{n}(s, y)+D_{r, \xi, z} u_{n}(s, y)\right)-\sigma\left(u_{n}(s, y)\right)\right] \\
\cdot L(d s, d y) .
\end{aligned}
$$

It remains to prove that

$$
A_{n+1}=\sup _{(t, x) \in[0, T] \times \mathbb{R}} E\left\|D u_{n+1}(t, x)\right\|_{\mathscr{H}}^{2}<\infty .
$$

Using (53), the isometry property (8), relation (44), and the fact that $\sigma$ is Lipschitz, we see that

$$
\begin{aligned}
& E\left|D_{r, \xi, z} u_{n+1}(t, x)\right|^{2} \leq 2 z^{2} G^{2}(t-r, x-\xi) \\
& \cdot E\left|\sigma\left(u_{n}(r, \xi)\right)\right|^{2}+2 v E \int_{0}^{t} \int_{\mathbb{R}} G^{2}(t-s, x-y) \\
& \cdot \mid \sigma\left(u_{n}(s, y)+D_{r, \xi, z} u_{n}(s, y)\right) \\
& -\left.\sigma\left(u_{n}(s, y)\right)\right|^{2} d y d s \leq 4 z^{2} D_{\sigma}^{2}(1 \\
& \left.+K_{n}\right) G^{2}(t-r, x-\xi) \\
& +2 v C_{\sigma}^{2} E \int_{0}^{t} \int_{\mathbb{R}} G^{2}(t-s, x-y) \\
& +\left|D_{r, \xi, z} u_{n}(s, y)\right|^{2} d y d s .
\end{aligned}
$$

We perform integration with respect to $d r d \xi \nu(d z)$ on $[0, T] \times$ $\mathbb{R} \times \mathbb{R}_{0}$. We denote

$$
v_{t}=\int_{0}^{t} \int_{\mathbb{R}} G^{2}(s, y) d y d s
$$

We obtain

$$
\begin{aligned}
E \| & D u_{n+1}(t, x) \|_{\mathscr{H}}^{2} \leq 4 v D_{\sigma}^{2}\left(1+K_{n}\right) v_{t} \\
& +2 v C_{\sigma}^{2} \int_{0}^{t} \int_{\mathbb{R}} G^{2}(t-s, x-y) \\
& +E\left\|D u_{n}(s, y)\right\|_{\mathscr{H}}^{2} d y d s \leq 4 v D_{\sigma}^{2}\left(1+K_{n}\right) v_{t} \\
& +2 v C_{\sigma}^{2} A_{n} v_{t} .
\end{aligned}
$$

Relation (54) follows taking the supremum over $(t, x) \epsilon$ $[0, T] \times \mathbb{R}$.

Step 2. We prove that $\sup _{n \geq 1} A_{n}<\infty$. By (57), we have

$$
\begin{aligned}
E\left\|D u_{n+1}(t, x)\right\|_{\mathscr{H}}^{2} \leq & 4 v D_{\sigma}^{2}\left(1+K_{n}\right) v_{t} \\
& +2 v C_{\sigma}^{2} \int_{0}^{t} V_{n}(s) J(t-s) d s,
\end{aligned}
$$

where $V_{n}(t)=\sup _{x \in \mathbb{R}} E\left\|D u_{n}(t, x)\right\|_{\mathscr{H}}^{2}$ and $J(t)$ is given by (33). This shows that

$$
\begin{aligned}
V_{n+1}(t) \leq & 4 v D_{\sigma}^{2} \nu_{T}(1+K) \\
& +2 v C_{\sigma}^{2} \int_{0}^{t} V_{n}(s) J(t-s) d s
\end{aligned}
$$

where $K$ is given by (37). By Lemma 15 of [16], $\sup _{n \geq 1} \sup _{t \in[0, T]} V_{n}(t)<\infty$. article.

We are now ready to state the main result of the present

Theorem 13. Assume that $\sigma$ is an affine function; that is, $\sigma(x)=a x+b$ for some $a, b \in \mathbb{R}$. If $u$ is the solution of (1), then, for any $t \in[0, T]$ and $x \in \mathbb{R}$,

$$
u(t, x) \in \mathbb{D}^{1,2}
$$

and the following relation holds in $L^{2}(\Omega ; \mathscr{H}$ ) (and hence, almost surely, for $\mu$-almost all $(r, \xi, z) \in U)$ :

$$
\begin{aligned}
D_{r, \xi, z} u(t, x)=G(t-r, x-\xi) \sigma(u(r, \xi)) z \\
\quad+\int_{0}^{t} \int_{\mathbb{R}} G(t-s, x-y) \\
\cdot\left[\sigma\left(u(s, y)+D_{r, \xi, z} u(s, y)\right)-\sigma(u(s, y))\right] \\
\cdot L(d s, d y) .
\end{aligned}
$$

Proof.

Step 1 . For any $t \in[0, T]$ and $n \geq 0$, let

$$
M_{n}(t)=\sup _{x \in \mathbb{R}} E\left\|D u_{n}(t, x)-D u_{n-1}(t, x)\right\|_{\mathscr{H}}^{2} .
$$

Note that, by Lemma $12, u_{n}(t, x) \in \mathbb{D}^{1,2}$ for any $(t, x) \in[0, T] \times$ $\mathbb{R}$ and $n \geq 0$.

Fix $(r, \xi, z) \in U$. We write relation (53) for $D_{r, \xi, z} u_{n+1}(t, x)$ and $D_{r, \xi, z} u_{n}(t, x)$. We take the difference between these two equations. We obtain

$$
\begin{aligned}
D_{r, \xi, z} u_{n+1}(t, x)-D_{r, \xi, z} u_{n}(t, x)=G(t-r, x-\xi) \\
\cdot\left[\sigma\left(u_{n}(r, \xi)\right)-\sigma\left(u_{n-1}(r, \xi)\right)\right] z+\int_{0}^{t} \int_{\mathbb{R}} G(t-s, x \\
-y)\left\{\left[\sigma\left(u_{n}(s, y)+D_{r, \xi, z} u_{n}(s, y)\right)-\sigma\left(u_{n}(s, y)\right)\right]\right. \\
\left.-\left[\sigma\left(u_{n-1}(s, y)+D_{r, \xi, z} u_{n-1}(s, y)\right)-\sigma\left(u_{n-1}(s, y)\right)\right]\right\} \\
\cdot L(d s, d y) .
\end{aligned}
$$

At this point, we use the assumption that $\sigma$ is the affine function $\sigma(x)=a x+b$. (An explanation of why this argument 
does not work in the general case is given in Remark 14.) In this case, relation (63) has the following simplified expression:

$$
\begin{aligned}
D_{r, \xi, z} u_{n+1}(t, x)-D_{r, \xi, z} u_{n}(t, x)=a G(t-r, x-\xi) \\
\cdot\left[u_{n}(r, \xi)-u_{n-1}(r, \xi)\right] z \\
\quad+a \int_{0}^{t} \int_{\mathbb{R}} G(t-s, x-y) \\
\cdot\left[D_{r, \xi, z} u_{n}(s, y)-D_{r, \xi, z} u_{n-1}(s, y)\right] L(d s, d y) .
\end{aligned}
$$

Using Itô's isometry and the inequality $(a+b)^{2} \leq 2\left(a^{2}+b^{2}\right)$, we obtain

$$
\begin{gathered}
E\left|D_{r, \xi, z} u_{n+1}(t, x)-D_{r, \xi, z} u_{n}(t, x)\right|^{2} \leq 2 a^{2} z^{2} G^{2}(t \\
-r, x-\xi) b_{n}^{2}+2 a^{2} v E \int_{0}^{t} \int_{\mathbb{R}} G^{2}(t-s, x-y) \\
\cdot\left|D_{r, \xi, z} u_{n}(s, y)-D_{r, \xi, z} u_{n-1}(s, y)\right|^{2} d y d s,
\end{gathered}
$$

where $b_{n}^{2}=\sup _{(s, y) \in[0, T] \times \mathbb{R}} E\left|u_{n}(s, y)-u_{n-1}(s, y)\right|^{2}$. Note that both sides of the previous inequality are zero if $r>t$. Taking the integral with respect to $d r d \xi \nu(d z)$ on $[0, T] \times \mathbb{R} \times \mathbb{R}_{0}$, we obtain

$$
\begin{aligned}
E & \left\|D u_{n+1}(t, x)-D u_{n}(t, x)\right\|_{\mathscr{H}}^{2} \leq 2 a^{2} v v_{t} b_{n}^{2} \\
& +2 a^{2} v E \int_{0}^{t} \int_{\mathbb{R}} G^{2}(t-s, x-y) \\
\cdot & E\left\|D u_{n}(s, y)-D u_{n-1}(s, y)\right\|_{\mathscr{H}}^{2} d y d s,
\end{aligned}
$$

where $v_{t}$ is given by (56). Recalling the definition of $M_{n}(t)$, we infer that

$$
M_{n+1}(t) \leq C_{n}+2 a^{2} v \int_{0}^{t} M_{n}(s) J(t-s) d s,
$$

where $C_{n}=2 a^{2} v v_{t} b_{n}^{2}$ and the function $J$ is given by (33). By relation (35), we know that $\sum_{n \geq 1} b_{n}<\infty$, which means that $\sum_{n \geq 1} C_{n}^{1 / 2}<\infty$. By Lemma A.1 (the Appendix), we conclude that

$$
\sum_{n \geq 1} \sup _{t \leq T} M_{n}(t)^{1 / 2}<\infty .
$$

Hence, the sequence $\left\{D u_{n}(t, x)\right\}_{n \geq 1}$ converges in $L^{2}(\Omega ; \mathscr{H})$ to a variable $U(t, x)$, uniformly in $(t, x) \in[0, T] \times \mathbb{R}$; that is,

$$
\sup _{(t, x) \in[0, T] \times \mathbb{R}} E\left\|D u_{n}(t, x)-U(t, x)\right\|_{\mathscr{H}}^{2} \longrightarrow 0 .
$$

Step 2. We fix $(t, x) \in[0, T] \times \mathbb{R}$. By $(36), u_{n}(t, x) \rightarrow u(t, x)$ in $L^{2}(\Omega)$. By Step 1, $\left\{D u_{n}(t, x)\right\}_{n \geq 1}$ converges in $L^{2}(\Omega ; \mathscr{H})$. We apply Theorem 4 to the variables $F_{n}=u_{n}(t, x)$ and $F=u(t, x)$. We infer that $u(t, x) \in \mathbb{D}^{1,2}$ and $D u_{n}(t, x) \rightarrow D u(t, x)$ in $L^{2}(\Omega ; \mathscr{H})$. Combining this with (69), we obtain

$$
\sup _{(t, x) \in[0, T] \times \mathbb{R}} E\left\|D u_{n}(t, x)-D u(t, x)\right\|_{\mathscr{H}}^{2} \longrightarrow 0 .
$$

Relation (61) follows by taking the limit in $L^{2}(\Omega ; \mathscr{H})$ as $n \rightarrow$ $\infty$ in (53).
Remark 14. Unfortunately, we were not able to extend Theorem 13 to an arbitrary Lipschitz function $\sigma$. To see where the difficulty comes from, recall that we need to prove that $\left\{D u_{n}(t, x)\right\}_{n \geq 1}$ converges in $L^{2}(\Omega ; \mathscr{H})$, and the difference $D_{r, \xi, z} u_{n+1}(t, x)-D_{r, \xi, z} u_{n}(t, x)$ is given by (63). For an arbitrary Lipschitz function $\sigma$, by relation (28), we have

$$
\begin{aligned}
\mid \sigma & \left(u_{n}(s, y)+D_{r, \xi, z} u_{n}(s, y)\right) \\
& -\sigma\left(u_{n-1}(s, y)+D_{r, \xi, z} u_{n-1}(s, y)\right) \mid \\
& \leq C_{\sigma} \mid\left(u_{n}(s, y)+D_{r, \xi, z} u_{n}(s, y)\right) \\
& -\left(u_{n-1}(s, y)+D_{r, \xi, z} u_{n-1}(s, y)\right) \mid \\
& \leq C_{\sigma}\left\{\left|u_{n}(s, y)-u_{n-1}(s, y)\right|\right. \\
& \left.+\left|D_{r, \xi, z} u_{n}(s, y)-D_{r, \xi, z} u_{n-1}(s, y)\right|\right\} .
\end{aligned}
$$

Using (63), the isometry property (8), the inequality ( $a+$ $b)^{2} \leq 2\left(a^{2}+b^{2}\right)$, and the previous inequality, we have

$$
\begin{aligned}
& E\left|D_{r, \xi, z} u_{n+1}(t, x)-D_{r, \xi, z} u_{n}(t, x)\right|^{2} \leq 2 z^{2} C_{\sigma}^{2} G^{2}(t \\
& \quad-r, x-\xi) E\left|u_{n}(r, \xi)-u_{n-1}(r, \xi)\right|^{2} \\
& \quad+4 v C_{\sigma}^{2} \int_{0}^{t} \int_{\mathbb{R}} G^{2}(t-s, x-y) \\
& \cdot E\left|u_{n}(s, y)-u_{n-1}(s, y)\right|^{2} d y d s \\
& \quad+4 v C_{\sigma}^{2} \int_{0}^{t} \int_{\mathbb{R}} G^{2}(t-s, x-y) \\
& \cdot E\left|D_{r, \xi, z} u_{n}(s, y)-D_{r, \xi, z} u_{n-1}(s, y)\right| d y d s .
\end{aligned}
$$

The problem is that the second term on the right-hand side of the inequality above does not depend on $(r, \xi, z)$ and hence its integral with respect to $d r d \xi \nu(d z)$ on $[0, T] \times \mathbb{R} \times \mathbb{R}_{0}$ is equal to $\infty$.

\section{Appendix}

\section{A Variant of Gronwall's Lemma}

The following result is a variant of Lemma 15 of [16], which is used in the proof of Theorem 13.

Lemma A.1. Let $\left(f_{n}\right)_{n \geq 0}$ be a sequence of nonnegative functions defined on $[0, T]$ such that $M=\sup _{t \in[0, T]} f_{0}(t)<\infty$ and, for any $t \in[0, T]$ and $n \geq 0$,

$$
f_{n+1}(t) \leq C_{n}+\int_{0}^{t} f_{n}(s) g(t-s) d s,
$$

where $g$ is a nonnegative function on $[0, T]$ with $\int_{0}^{T} g(t) d t<$ $\infty$ and $\left(C_{n}\right)_{n \geq 0}$ is a sequence of nonnegative constants. Then, there exists a sequence $\left(a_{n}\right)_{n \geq 0}$ of nonnegative constants which 
satisfy $\sum_{n \geq 0} a_{n}^{1 / p}<\infty$ for any $p>1$, such that, for any $t \in$ $[0, T]$ and $n \geq 0$,

$$
f_{n}(t) \leq C_{n}+\sum_{j=1}^{n-1} C_{j} a_{n-j}+C_{0} a_{n} M .
$$

In particular, if $\sum_{n \geq 1} C_{n}^{1 / p}<\infty$ for some $p>1$, then

$$
\sum_{n \geq 1} \sup _{t \in[0, T]} f_{n}(t)^{1 / p}<\infty
$$

Proof. Let $G(T)=\int_{0}^{T} g(t) d t,\left(X_{i}\right)_{i \geq 1}$ be a sequence of i.i.d. random variables on $[0, T]$ with density function $g(t) / G(T)$, and $S_{n}=\sum_{i=1}^{n} X_{i}$. Following exactly the same argument as in the proof of Lemma 15 of [16], we have

$$
\begin{aligned}
f_{n}(t) \leq & C_{n}+C_{n-1} G(T) P\left(S_{1} \leq t\right)+\cdots \\
& +C_{1} G(T)^{n-1} P\left(S_{n-1} \leq t\right) \\
& +C_{0} G(T)^{n} E\left[1_{\left\{S_{n} \leq t\right\}} f_{0}\left(t-S_{n}\right)\right] .
\end{aligned}
$$

Relation (A.2) follows with $a_{n}=G(T)^{n} P\left(S_{n} \leq T\right)$ for $n \geq 1$. The fact that $\sum_{n \geq 1} a_{n}^{1 / p}<\infty$ for all $p \geq 1$ was shown in the proof of Lemma 17 of [16].

To prove the last statement, we let $a_{0}=1$ and $M_{1}=$ $\max (M, 1)$. Then, $f_{n}(t) \leq M_{1} \sum_{j=0}^{n} C_{j} a_{n-j}$ and, hence, $\sup _{t \leq T} f_{n}(t)^{1 / p} \leq M_{1}^{1 / p} \sum_{j=0}^{n} C_{j}^{1 / p} a_{n-j}^{1 / p}$. We conclude that

$$
\begin{aligned}
\sum_{k=0}^{n} \sup _{t \leq T} f_{k}(t)^{1 / p} & \leq M_{1}^{1 / p} \sum_{j=0}^{n} C_{j}^{1 / p} \sum_{k=j}^{n} a_{k-j}^{1 / p} \\
& \leq M_{1}^{1 / p} \sum_{j \geq 0} C_{j}^{1 / p} \sum_{k \geq 0} a_{k}^{1 / p}:=C<\infty .
\end{aligned}
$$

\section{Conflicts of Interest}

The authors declare that there are no conflicts of interest regarding the publication of this paper.

\section{Acknowledgments}

This research supported by a grant from the Natural Sciences and Engineering Research Council of Canada.

\section{References}

[1] J. B. Walsh, "An introduction to stochastic partial differential equations," in École d'Été de Probabilités de Saint Flour XIV1984, vol. 1180 of Lecture Notes in Math., pp. 265-439, Springer, Heidelberg, Germany, 1986.

[2] R. Dalang, D. Khoshnevisan, C. Mueller, D. Nualart, and Y. Xiao, A minicourse on stochastic partial differential equations, vol. 1962 of Lecture Notes in Mathematics, Springer, Heidelberg, Berlin, 2009.
[3] D. Khoshnevisan, Analysis of Stochastic Partial Differential Equations, vol. 119 of CBMS Regional Conference Series in Mathematics, AMS, Providence, RI, USA, 2014.

[4] D. Nualart, Malliavin Calculus, Springer, 2nd edition, 2006.

[5] M. Sanz-Solé, Malliavin Calculus with Applications to Stochastic Partial Differential Equations, EPFL Press, CRC Press, Boca Raton, Fla, USA, 2005.

[6] D. Applebaum, Lévy Processes and Stochastic Calculus, vol. 116 of Cambridge Studies in Advanced Mathematics, Cambridge University Press, Cambridge, UK, 2nd edition, 2009.

[7] K. Bichteler, J.-B. Gravereaux, and J. Jacod, Malliavin Calculus for Processes with Jumps, vol. 2 of Stochastics Monographs, Gordon and Breach Science, New York, NY, USA, 1987.

[8] G. Di Nunno, B. Oksendal, and F. Proske, Malliavin Calculus for Lévy Processes with Applications to Finance, Universitext, Springer, Berlin, Germany, 2009.

[9] G. Da Prato and J. Zabczyk, Stochastic Equations in Infinite Dimensions, Cambridge University Press, Cambridge, UK, 1992.

[10] R. C. Dalang and L. s. Quer-Sardanyons, "Stochastic integrals for spde's: a comparison," Expositiones Mathematicae, vol. 29, no. 1, pp. 67-109, 2011.

[11] S. Peszat and J. Zabczyk, Stochastic Partial Differential Equations with Lévy Noise, vol. 113 of Encyclopedia of Mathematics and its Applications, Cambridge University Press, Cambridge, UK, 2007.

[12] R. M. Balan, "Integration with respect to Lévy colored noise, with applications to SPDEs," Stochastics, vol. 87, no. 3, pp. 363381, 2015.

[13] C. B. Ndongo, Equations aux dérivées partielles stochastiques avec bruit blanc de Lévy [Ph.D. thesis], University of Ottawa, 2016 (French).

[14] R. M. Balan and C. B. Ndongo, "Ito formula for integral processes related to space-time Lévy noise," Applied Mathematics, vol. 6, no. 10, pp. 1755-1768, 2015.

[15] R. M. Balan and C. B. Ndongo, "Intermittency for the wave equation with Lévy white noise," Statistics \& Probability Letters, vol. 109, pp. 214-223, 2016.

[16] R. C. Dalang, "Extending martingale measure stochastic integral with applications to spatially homogeneous s.p.d.e.s," Electronic Journal of Probability, vol. 4, no. 6, 29 pages, 1999, Erratum in Electronic Journal of Probability, vol. 6, 5 pages, 2001. 


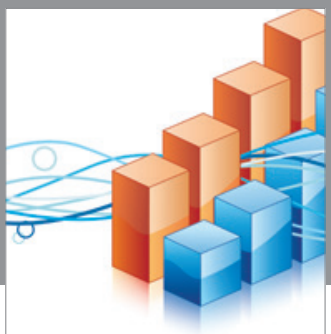

Advances in

Operations Research

vatem alat4

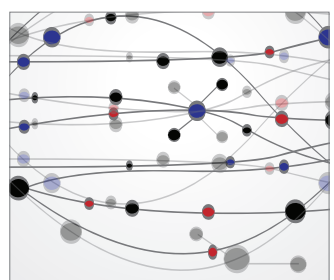

\section{The Scientific} World Journal
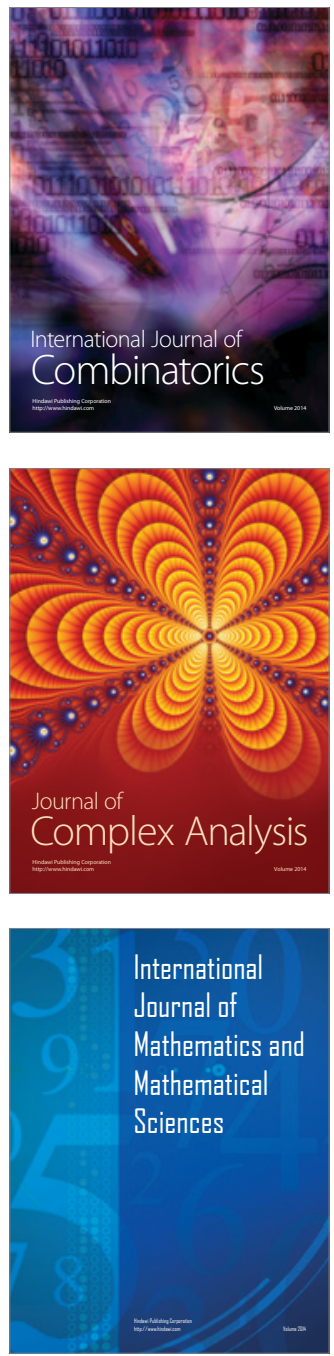
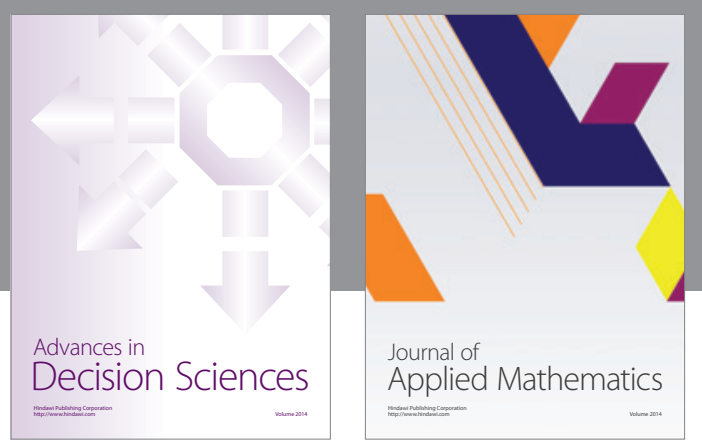

Algebra

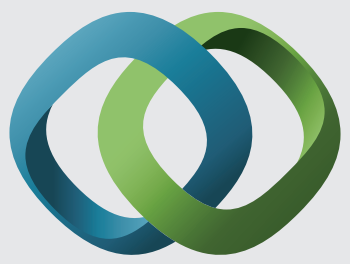

\section{Hindawi}

Submit your manuscripts at

https://www.hindawi.com
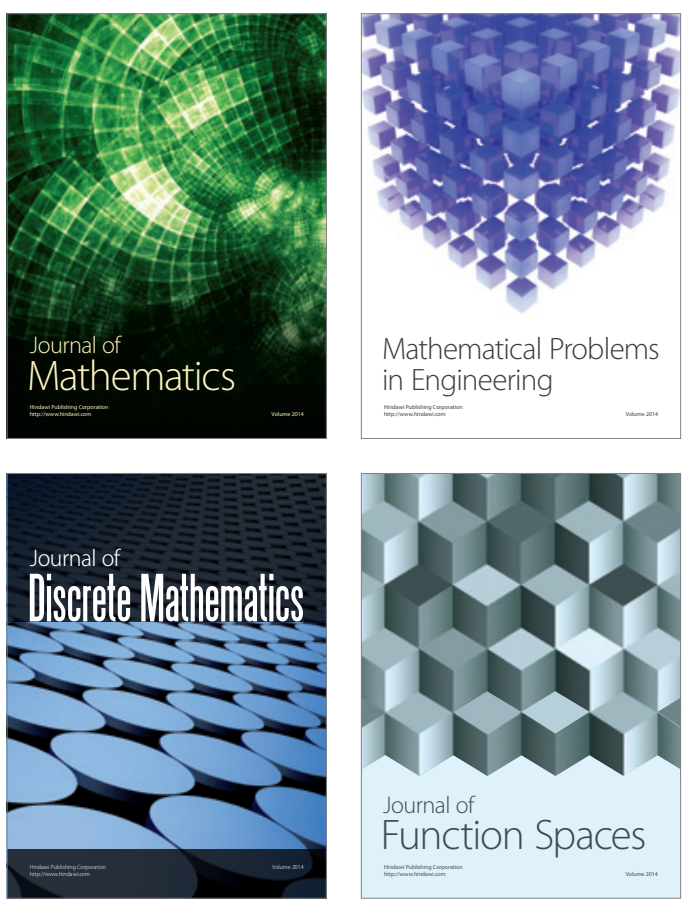

Mathematical Problems in Engineering
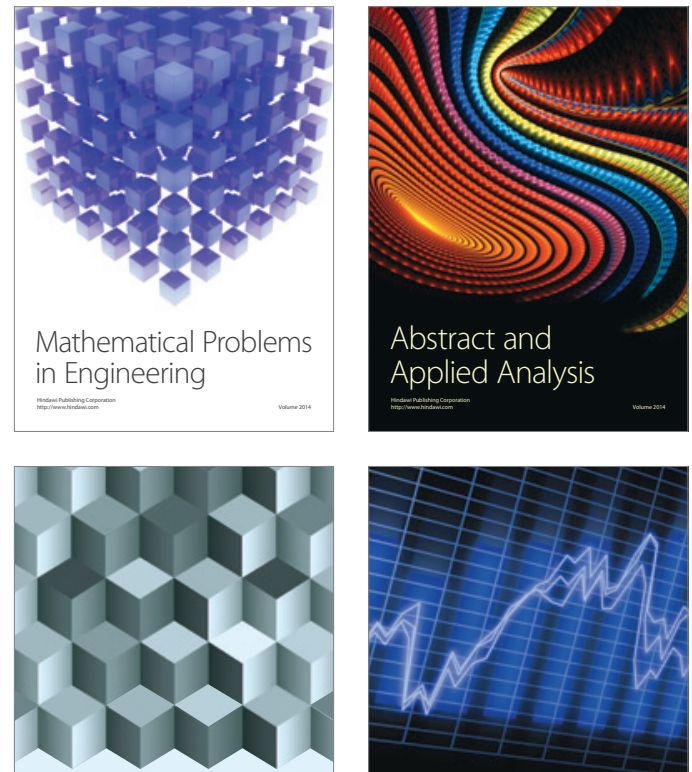

Journal of

Function Spaces

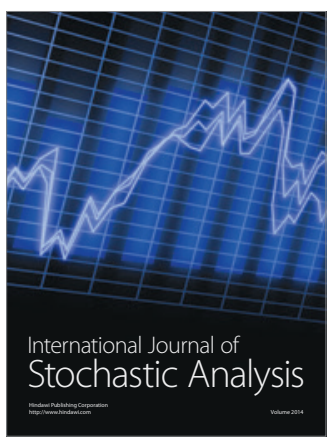

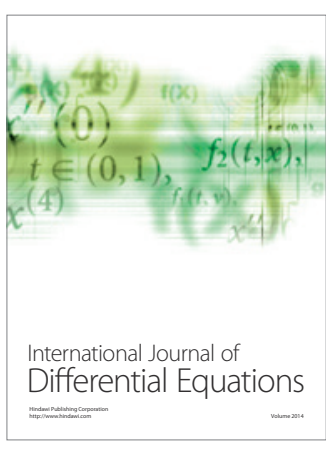
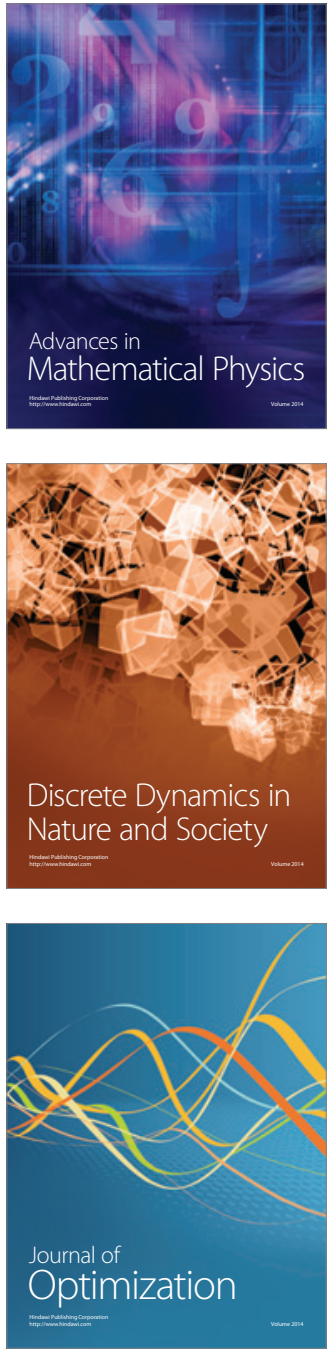